\title{
Revisiting Holographic Dark Energy in Cyclic Cosmology
}

\author{
A. Sheykhi ${ }^{1,2 *}$, M. Tavayef ${ }^{1}$, H. Moradpour ${ }^{2 \dagger}$ \\ ${ }^{1}$ Physics Department and Biruni Observatory, College of Sciences, Shiraz University, Shiraz 71454, Iran \\ ${ }^{2}$ Research Institute for Astronomy and Astrophysics of Maragha (RIAAM), P.O. Box 55134-441, Maragha, Iran
}

\begin{abstract}
Considering the holographic dark energy (HDE) with two different Infrared (IR) cutoffs, we study the evolution of a cyclic universe which avoids the Big-Rip singularity. Our results show that, even in the absence of a mutual interaction between the cosmos sectors, the HDE model with the Hubble radius as IR cutoff can mimics a cosmological constant in the framework of a cyclic cosmology. In addition, we find that both the interacting and non-interacting universes may enter into a cycle of sequential contraction and expansion, if the Granda-Oliveros (GO) cutoff is chosen as the IR cutoff in the energy density of the HDE.
\end{abstract}

\section{INTRODUCTION}

There are a lot of observations data which signal us to an accelerating expanding universe [1-3]. In order to describe this current phase of the universe expansion, we need a strange source of energy which is called dark energy and allocates approximately $73 \%$ of the cosmos tissue to itself. WMAP observations also indicates that the pressure $(p)$ and energy density $(\rho)$ of dark energy are bounded to $p / \rho \simeq-1.10$, a result addressing a phantom type dark energy [3]. Therefore, in addition to the initial Big Bang singularity, another singularity called Big Rip, can be achieved by cosmos [4 6]. It was argued that a cyclic universe may avoid these singularities [7, 8].

In a cyclic cosmology, which has firstly been introduced by Tolman [9], our Universe successive expands and contracts. A new version of cyclic model in which the universe cycle is realized in the light of two separated branes, has been proposed by Steinhardt et al. [10]. Although, this new model gives us an alternative to the inflation scenario, it suffers from two problems including the entropy and black hole problems [7, 8, 11]. It is finally useful to note here that these problems can be solved by considering some features of phantom dark energy [7, 8].

A cyclic universe is supported by an enormously high energy density at the beginning and end of a cycle meaning that the phantom dark energy increases more than expected in a finite time [4, 12]. As a result of this behavior, one may expect that the high energy physics induces some modifications to the Friedmann equation. Recently, such modifications have been proposed arisen from braneworld scenario [13], and Loop Quantum Cosmology (LQC) [14, 15].

In LQC, which enumerates the quantum nature of spacetime [14], the Friedman equation is modified as

$$
H^{2}=\frac{\rho}{3 m_{p}^{2}}\left(1-\frac{\rho}{\rho_{c}}\right)
$$

where $\rho$ is the total energy density of fluids which support the background. Moreover, $H=\dot{a} / a$ and $m_{p}$

\footnotetext{
*asheykhi@shirazu.ac.ir

†h.moradpour@riaam.ac.ir
}

are the Hubble parameter and the reduced planck mass $\left(m_{p}^{2}=1 / 8 \pi G=2.44 \times 10^{18} \mathrm{Gev}\right)$, respectively. $\rho_{c}=$ $4 \sqrt{3} \lambda^{-3} m_{p}^{4}=0.82 \rho_{p}$, where $\rho_{p}=2.22 \times 10^{76} \mathrm{GeV}$, denotes the critical energy density set by quantum gravity and differs from the usual critical density $\left(3 m_{p}^{2} H^{2}\right)$. At the $\rho_{c}$ point, called turnaround point, universe begins to collapse and by doing this, it avoids the Big-Rip singularity. The universe contraction continues until it reaches at the bounce point, where the universe starts to expand again. It is worth mentioning that, at the $\rho \ll \rho_{c}$ limit, the term $\rho / \rho_{c}$ in Eq. (1) is negligible and thus the Friedmann equation is obtainable.

There is a novel proposal for dark energy, which comes from holographic hypothesis [16, 17], and is called holographic dark energy (HDE) [18, 19]. This proposal is based on the Cohen et al, idea where they suggest the $L^{3} \Lambda^{4} \leq L_{m_{p}^{2}}$ relation between the ultraviolet and IR cutoffs denoted as $\Lambda$ and $L$, respectively [18]. Applying this idea to cosmos, one can get the holographic dark energy density as

$$
\rho_{D}=3 c^{2} m_{p}^{2} L^{-2}
$$

where $3 c^{2}$ is a constant, and its value can be determined by observations, and $L$ is a length which is related to the size of the universe. A primary option for $L$ is the Hubble parameter which does not lead to acceptable result in the framework of standard cosmology [20]. Assuming the particle horizon as IR cutoff, the accelerated expansion cannot be achieved, too [19]. To achieve an accelerated universe, Li suggested that one should take the future event horizon of our universe as the proper IR cutoff, in order to produce a correct equation of state for HDE [19]. For a comprehensive list of references concerning HDE, we refer to [21 30] and references therein. The HDE model have also been constrained and tested by various astronomical observations $31-34$.

Furthermore, Granda and Oliveros have proposed another cutoff which is a generalization of Ricci cutoff and is known as the GO cutoff [35]. Some cosmological implications of the HDE model with GO cutoff have been explored in [36 39].

On the other hand, observations admit a mutual interaction between the dark sectors of the universe [39 48]. 
Motivated by these observations, various kind of interactions have been proposed and studied in various cosmological theories [49 53]. Finally, it is worth mentioning that such interaction may solve the coincidence problem [54 60].

The studies on the HDE models with various cutoff were also extended to a cyclic universe. In this regards, the cosmological evolution of HDE model with the future event horizon radius as IR cutoff have been investigated in a cyclic universe [61]. Also, interacting new agegraphic dark energy in a cyclic universe were explored in 62]. It was shown that when there is no interaction between dark matter and dark energy, agegraphic dark energy and new agegraphic dark energy do not produce a phantom phase, and therefore cannot describe a cyclic universe [62]. Furthermore, the ghost dark energy and the generalized ghost dark energy in the framework of cyclic cosmology were explored, recently [63]. It was argued that in the absence of interaction in a cyclic universe, the deceleration parameter becomes a constant; as a result the universe cannot move from an accelerated expansion phase to a deceleration phase and so cannot reach to turnaround point and start to contract 63]. It was also argued that only in the presence of interaction, the transition from an accelerated expansion phase to a deceleration phase in the future near the (turnaround point) can be achieved in a cyclic universe 63.

In the present paper, we are interested in studying the cosmological consequences of the HDE hypothesis in the framework of cyclic universe. It is noteworthy to mention that our work differs from [35] in that, they studied HDE model with GO cutoff in standard cosmology, while we investigate this model in a cyclic universe. In order to achieve this goal, we focus on the Hubble and GO cutoffs, and study the evolution of the universe. The structure of this paper is as follows. In the next section, we consider a cyclic universe filled with HDE and dark matter. As system's IR cutoff we shall take the Hubble radius. In section III, we study the HDE model in a cyclic universe with GO cutoff. The last section is devoted to summary and concluding remarks.

\section{HDE IN A CYCLIC UNIVERSE}

\section{A. Non-interacting case}

For a flat FRW universe filled by a pressureless matter and a dark energy component, the Friedmann equation is

$$
H^{2}=\frac{\rho_{m}+\rho_{D}}{3 m_{p}^{2}}\left[1-\left(\frac{\rho_{m}+\rho_{D}}{\rho_{c}}\right)\right],
$$

where $\rho_{m}$ is the energy density of the pressureless matter. In addition, when the cosmos sectors do not interact with each other, the continuity equation leads to

$$
\dot{\rho}_{m}+3 H \rho_{m}=0,
$$

$$
\dot{\rho}_{D}+3 H \rho_{D}\left(1+\omega_{D}\right)=0,
$$

where $\omega_{D}=p_{D} / \rho_{D}$ and $p_{D}$ denote the state parameter (EoS) and pressure of DE, respectively. Dot is also the derivative with respect to cosmic time. We finally define the dimensionless density parameters as $\Omega_{m}=\rho_{m} /\left(3 m_{p}^{2} H^{2}\right)$ and $\Omega_{D}=\rho_{D} /\left(3 m_{p}^{2} H^{2}\right)$. Now, inserting $u=\Omega_{m} / \Omega_{D}$ into Eq. (3), we obtain

$$
1-\frac{2 \rho}{\rho_{c}}=\frac{2-\Omega_{D}(1+u)}{\Omega_{D}(1+u)} .
$$

Considering the Hubble radius $L=H^{-1}$ as the IR cutoff, and using Eq. (2), one gets

$$
\rho_{D}=3 c^{2} m_{p}^{2} L^{-2}=3 c^{2} m_{p}^{2} H^{2},
$$

and the density parameter becomes $\Omega_{D}=c^{2}$. Combining Eqs. (5) and (7), one arrives at

$$
\omega_{D}=-\frac{2}{3} \frac{\dot{H}}{H^{2}}-1
$$

On the other hand, using Eqs. (11), (4) and (5), it is a matter of calculation to show that

$$
\frac{\dot{H}}{H^{2}}=-\frac{3}{2} \Omega_{D}\left(1+u+\omega_{D}\right)\left(1-\frac{2 \rho}{\rho_{c}}\right) .
$$

Substituting this relation into Eq. ([6), one gets

$$
\omega_{D}=\left[1-\Omega_{D}(1+u)\right]\left(\frac{1+u}{(1+u)\left(1+\Omega_{D}\right)-2}\right) .
$$

for the EoS of HDE in a non-interacting universe. It is worth mentioning that, at the high energy limit, where $\Omega_{m} \ll \Omega_{D}$ and thus $u \approx 0$, this equation leads to

$$
\omega_{D} \approx-1 \text {. }
$$

Indeed, for $u>0$, we always have $\omega_{D}<-1$, which implies that a HDE with Hubble radius as IR cutoff, in a cyclic universe, behaves as the phantom source. This result is clearly in contrast to the non-interacting HDE model in standard cosmology where $\omega_{D}=0$ for $L=H^{-1}$ [19]. Bearing in mind the definition of deceleration parameter as

$$
q=-1-\frac{\dot{H}}{H^{2}},
$$

and using Eqs. (6), (9) and (10) to evaluate the deceleration parameter, after simple calculations, one finds

$$
q=-1+\frac{3 u\left(2-\Omega_{D}(1+u)\right)}{2\left[(1+u)\left(1+\Omega_{D}\right)-2\right]} .
$$

This implies that $q \approx-1$ at the $u \approx 0$ limit. This asymptotic behavior is an interesting result, because in the original hypothesis of HDE, this IR cutoff leads to $q=1 / 2$ [19]. Indeed, since $q \leq-1$ for $u \geq 0$, the accelerated phase of the universe expansion does not stop in this model. 


\section{B. Interacting case}

When the cosmos sectors interact with each other through a mutual interaction $Q$, the energy-momentum conservation law is decomposed as

$$
\begin{gathered}
\dot{\rho}_{m}+3 H \rho_{m}=Q, \\
\dot{\rho}_{D}+3 H \rho_{D}\left(1+\omega_{D}\right)=-Q .
\end{gathered}
$$

Throughout this paper, we consider an interaction term as [39, 54]

$$
Q=3 b^{2} H \rho=3 b^{2} H\left(\rho_{D}+\rho_{m}\right)=3 b^{2} H \rho_{D}(1+u),
$$

where $b$ is the coupling constant. Employing this equation as well as Eqs. (7) and (15), one obtains

$$
\omega_{D}=-1-\frac{2}{3} \frac{\dot{H}}{H^{2}}-b^{2}(1+u)
$$

and

$$
\frac{\dot{H}}{H^{2}}=-\frac{3}{2} \Omega_{D}\left(1+u+\omega_{D}\right)\left(1-\frac{2 \rho}{\rho_{c}}\right) .
$$

wherein we combined Eqs. (3), (14) and (15) with Eq. (17) to get the last equation. One can also use Eqs. (6), (17) and (18) in order to show that

$$
\frac{\dot{H}}{H^{2}}=-\frac{3}{2} \Omega_{D}\left(1+u+\omega_{D}\right)\left(\frac{2-\Omega_{D}(1+u)}{\Omega_{D}(1+u)}\right),
$$

and

$$
\omega_{D}=\frac{(1+u)\left[1-\left(\Omega_{D}+b^{2}\right)(1+u)\right]}{(1+u)\left(1+\Omega_{D}\right)-2}
$$

At the $u \approx 0$ limit, where $\Omega_{D} \approx c^{2}$, we have

$$
\omega_{D} \approx-1-\frac{b^{2}}{c^{2}-1}
$$

which is constant and smaller than -1 . Therefore, this model predicts a constant value for $\omega_{D}$ at the current stage of the universe expansion, a result which is in conflict with the recent observations which indicates that the DE candidate should have a time varying EoS parameter [64]. Finally, calculations for the deceleration parameter lead to

$q=-1+\frac{3}{2}\left(2-\Omega_{D}(1+u)\right)\left[1+\frac{1-\left(\Omega_{D}+b^{2}\right)(1+u)}{(1+u)\left(1+\Omega_{D}\right)-2}\right]$

which finally yields

$$
q=2-\frac{3}{2} \Omega_{D}+\frac{3}{2} \frac{\left(2-\Omega_{D}\right)\left(1-\Omega_{D}-b^{2}\right)}{\Omega_{D}-1},
$$

for $u \approx 0$, and thus the constant value of

$$
q \approx \frac{\left(3 b^{2}-2\right) c^{2}-6 b^{2}+2}{2\left(c^{2}-1\right)}
$$

where we have used $\Omega_{D}=c^{2}$. If $1<c^{2}<\frac{6 b^{2}-1}{3 b^{2}-2}$ and $b^{2}>\frac{2}{3}$, then the deceleration parameter is negative, and therefore, our Universe experiences an endless accelerating expanding phase meaning that the turnaround point is not achieved by our Universe.

\section{HDE IN A CYCLIC UNIVERSE WITH GO CUTOFF}

Here, we consider the HDE model in a cyclic universe with GO cutoff which has firstly been introduced by Granda and Oliveros as 35]

$$
L=\left(\alpha^{\prime} H^{2}+\beta^{\prime} \dot{H}\right)^{-1 / 2},
$$

where $\alpha^{\prime}$ and $\beta^{\prime}$ are constants evaluated by other parts of physics as well as observations. It is in fact the formal generalization of the Ricci scalar curvature cutoff [38], and a HDE model with this cutoff may avoid the causality problem [65]. With this choice for cutoff, the energy density (2) becomes

$$
\rho_{D}=3 m_{p}^{2}\left(\alpha H^{2}+\beta \dot{H}\right)
$$

where $\alpha=c^{2} \alpha^{\prime}$ and $\beta=\beta^{\prime} c^{2}$, and the dimensionless density parameter takes the form

$$
\Omega_{D}=\frac{\rho_{D}}{3 m_{p}^{2} H^{2}}=\alpha+\beta \frac{\dot{H}}{H^{2}} .
$$

Finally, it is useful to note that since $c^{2}, \alpha$ and $\beta$ are unknown coefficients, the GO cutoff can be redefined as

$$
L=\left(\alpha H^{2}+\beta \dot{H}\right)^{-1 / 2},
$$

reduced to the Ricci cutoff $\left(L^{-2}=2 H^{2}+\dot{H}\right)$ for $\alpha=2$ and $\beta=1$ [38].

\section{A. Non-interacting case}

For a non-interacting universe, substituting Eq. (9) into Eq. (27) and using Eq. (6), one reaches at

$$
\omega_{D}=\left[\frac{2}{3 \beta}\left(\frac{\alpha-\Omega_{D}}{2-\Omega_{D}(1+u)}\right)-1\right](1+u) .
$$

In the high energy regime, where $u \approx 0$, this equation leads to

$$
\omega_{D} \approx \frac{2}{3 \beta}\left(\frac{\alpha-\Omega_{D}}{2-\Omega_{D}}\right)-1
$$

which states that for $\alpha=2$ the Eos parameter, is constant at this limit, namely $\omega_{D}=-1+2 / 3 \beta$. In this manner, in order to have a phantom source required for a cyclic universe $\left(\omega_{D}<-1\right)$, we should have $\beta<0$. Considering the high energy limit where HDE dominates 


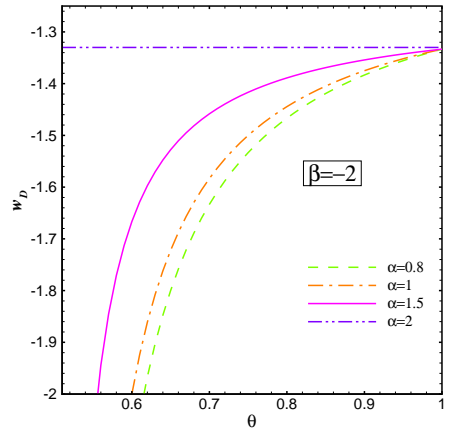

FIG. 1: The evolution of $\omega_{D}$ versus the dimensionless quantity $\theta=\rho_{D} / \rho_{c}$, for different values of $\alpha$ and $\beta=-2$.

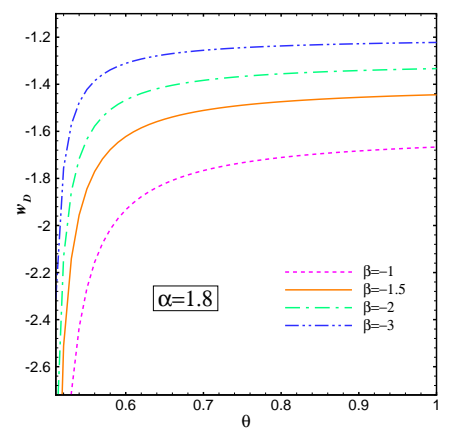

FIG. 2: The plot depicts $\omega_{D}$ as a function of $\theta$, for different values of $\beta$, when $\alpha=1 \cdot 8$.

$(u \approx 0)$. In this case we can neglect $\rho_{m}$ in the total energy density and the Friedmann equation (3) reduces to

$$
3 m_{p}^{2} H^{2}=\rho_{D}\left(1-\frac{\rho_{D}}{\rho_{c}}\right) .
$$

which can also be rewritten as

$$
\Omega_{D}=1+\frac{\rho_{D}}{\rho_{c}-\rho_{D}},
$$

Inserting $\theta=\rho_{D} / \rho_{c}$ into Eq. (32), one can easily obtains

$$
\Omega_{D}=1+\frac{\theta}{1-\theta}=\frac{1}{1-\theta},
$$

Combining with Eq. (30), we arrive at

$$
\omega_{D}=-1+\frac{2}{3 \beta}\left(\frac{-\alpha \theta+\alpha-1}{-2 \theta+1}\right)
$$

at the high energy limit. Therefore, for the Ricci cutoff, where $\alpha=2$ and $\beta=1$, we have $\omega_{D}=1 / 2$. In the low energy regime in which $\rho \ll \rho_{c}$ and therefore $3 H^{2}=\rho$ meaning that the usual Friedmann equation is obtainable, we have

$$
1+u=\frac{1}{\Omega_{D}}
$$

where we have used Eq. (6) in order to get this equation. Let us study the behavior of EoS parameter (29) at this limit, where the usual Friedmann equation is available and $\Omega_{D}=1$ [35]. For this propose, we substitute the above results into Eq. (29) to obtain

$$
\omega_{D}=-1+\frac{2(\alpha-1)}{3 \beta} .
$$

This is exactly the result obtained in [35] for the EoS parameter of HDE with GO cutoff in standard cosmology.

In Fig. 1 the evolution of the EoS parameter, at high energy limit, is plotted as a function of $\theta$ for $\beta=-2$ and $\alpha=0 \cdot 8,1,1 \cdot 5$ and 2 . As it is obvious, all diagrams converge to $\omega_{D} \simeq-1 \cdot 33$ at turnaround point, and in fact, HDE behaves as the phantom source for which $\omega_{D}<$ -1 . To complete the discussion, the EoS parameter as a function of $\theta$ has also been plotted for $\alpha=1 \cdot 8$ and different values of $\beta$ in Fig. 2 showing the effects of $\beta$ on EoS parameter in high energy regime.

Now, using $\Omega_{D}^{\prime}=d \Omega_{D} / d(\ln a)=\dot{\Omega}_{D} / H$ as well as Eq. (27), we obtain

$$
\Omega_{D}^{\prime}=\Omega_{D}\left(\frac{\dot{\rho_{D}}}{H \rho_{D}}-\frac{2 \dot{H}}{H^{2}}\right) .
$$

Additionally, one can combine Eqs. (5), (9) and the above relation with Eqs. (29) and (6) to get the following equation

$$
\Omega_{D}^{\prime}=3 \Omega_{D}\left[u+\frac{2\left(\alpha-\Omega_{D}\right)}{3 \beta}\left(1-\frac{1+u}{2-\Omega_{D}(1+u)}\right)\right],
$$

which finally leads to

$$
\Omega_{D}^{\prime}=\frac{2}{\beta} \Omega_{D}\left(1-\Omega_{D}\right)\left(\frac{\alpha-\Omega_{D}}{2-\Omega_{D}}\right),
$$

at the $u \approx 0$ limit. Bearing Eqs. (9) and (12) in mind, and using Eqs. (6) and (29), we reach at

$$
q=-1+\frac{\alpha-\Omega_{D}}{\beta},
$$

for the deceleration parameter. As we have already discussed, in order to satisfy the phantom dark energy condition i.e. $\omega_{D}<-1$, we should have $\beta<0$. In this case, an accelerating universe $(q<0)$ is achieved for $\Omega_{D}<\alpha-\beta$. Besides, whenever $\Omega_{D}=\alpha-\beta$, we have $q=0$ meaning that a transition from an accelerated expansion phase to a decelerated one happens. In Fig. 3. the deceleration parameter as a function of $\theta$ is plotted for various values of $\beta$ when $\alpha=1$.8. The negative values of $q$ indicates that universe experiences an accelerated expansion. Through the time and by increasing the HDE phantom density, $q$ will become larger, until it approaches to zero from below. Consequently, the universe moves from an accelerated phase to a decelerating phase. It is worth mentioning that this transition will earlier happen 


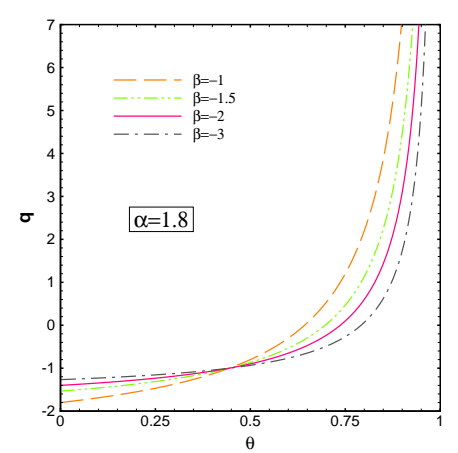

FIG. 3: The evolution of the deceleration parameter versus $\theta$ for various values of $\beta$ whenever $\alpha=1 \cdot 8$.

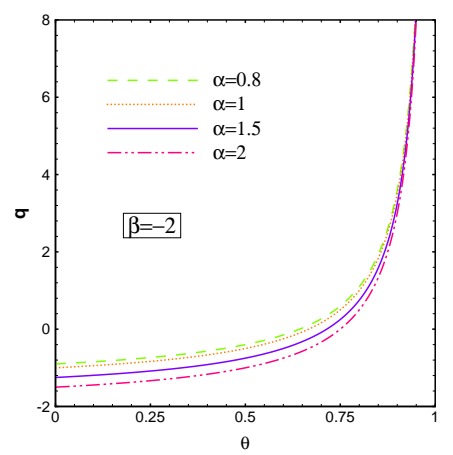

FIG. 4: The plot depicts $q$ as a function of $\theta$, when $\beta=-2$, for some values of $\alpha$.

for smaller values of $\beta$. Moreover, when the energy density approaches to the critical energy density $(\theta \rightarrow 1)$, the universe changes its evolution direction. We also plotted Fig. 4 to investigate the effects of $\alpha$ on $q$ showing that the larger values of $\alpha$ leads to more negative values for $q$, and therefore, the foresaid transition will happen later.

\section{B. Interacting case}

For an interacting universe, similar to the case of Hubble radius cutoff in the previous section, taking the time derivative of Eq. (11), and combining the result with Eqs. (14) and (15), we get Eq. (18). Now, inserting this relation into Eq. (27) and using Eq. (6), one obtains

$$
\omega_{D}=(1+u)\left[\frac{2}{3 \beta}\left(\frac{\alpha-\Omega_{D}}{2-\Omega_{D}(1+u)}\right)-1\right]
$$

which is the same as Eq. (29) in the non-interacting case. Finally, for $u \approx 0$ limit, the above relation reduces to

$$
\omega_{D}=-1+\frac{2}{3 \beta}\left(\frac{\alpha-\Omega_{D}}{2-\Omega_{D}}\right)
$$

On the other hand, combining Eqs. (15) and (19) with Eq. (37), and inserting the result into Eq. (41), we find

$$
\begin{aligned}
\Omega_{D}^{\prime} & =3 \Omega_{D}\left\{u-b^{2}(1+u)\right. \\
& \left.+\frac{2\left(\alpha-\Omega_{D}\right)}{3 \beta}\left(1-\frac{1+u}{2-\Omega_{D}(1+u)}\right)\right\} .
\end{aligned}
$$

In the absence of interaction $\left(b^{2}=0\right)$, our previous result obtained in Eq. (38) is recovered. Moreover, in high energy regime where $u=0$, one gets

$$
\Omega_{D}^{\prime}=-3 b^{2} \Omega_{D}+\frac{2}{\beta} \Omega_{D}\left(1-\Omega_{D}\right)\left(\frac{\alpha-\Omega_{D}}{2-\Omega_{D}}\right) .
$$

In order to achieve $q$, bearing Eq. (12) in mind, and substituting Eq. (19) into Eq. (41), one can easily show that

$$
q=-1+\frac{\alpha-\Omega_{D}}{\beta} .
$$

Therefore, unlike the evolution of the energy density parameter Eq. (44), the deceleration parameter of $\mathrm{HDE}$ model in a cyclic universe is independent of the coupling constant $b^{2}$.

\section{CONCLUDING REMARKS}

We have revisited the HDE model in the framework of cyclic cosmology by taking into account the Hubble radius as IR cutoff. For a non-interacting universe, our study shows that, at the high energy regime where $u=\rho_{m} / \rho_{D} \approx 0$, both the EoS parameter as well as the deceleration parameter of the HDE model in a cyclic universe approach to -1 . This implies that the acceleration of the universe expansion can be achieved in this model. Clearly, this result is in contrast to the HDE with Hubble cutoff in standard cosmology where its EoS parameter behaves like a dust, i.e., $\omega_{D}=0$, and hence cannot produce the accelerating universe. We also considered the interacting HDE model with Hubble radius as IR cutoff in the framework of cyclic cosmology and found that in this case the EoS parameter crosses the phantom divide, namely $\omega_{D}<-1$. It is worth noting that in both of these model $\omega_{D}$ becomes a constant value at the current stage of the universe expansion. This result is in conflict with some recent observations which indicates that the DE candidate should have a time varying EoS parameter 64.

We also studied the HDE with GO cutoff in the framework of cyclic universe. We found that this model supports a noninteracting accelerating universe in the high energy limit. In this manner, HDE behaves as a phantom source, and the universe expansion phase will change from an accelerating phase to a deceleration one. Finally, we found out that, for an interacting HDE with GO cutoff, the mutual interaction between the dark sectors of 
cosmos only affects the dimensionless density parameter of HDE, and it does not affect the deceleration parameter meaning that the behavior of $q$ in a noninteracting universe is the same as that of the interacting universe.

\section{Acknowledgments}

We thank Shiraz University Research Council. This work has been supported financially by Research Institute for Astronomy \& Astrophysics of Maragha (RIAAM), Iran.
[1] S. Perlmutter et al., Astrophys. J. 517, 565 (1999).

[2] A. G. Riess et al., Astron. J. 116, 1009 (1999).

[3] E. Kamatsu et al., Astrophys. J. Suppl. Ser. 192, 18 (2011).

[4] R. R. Caldwell, M. kamionkowski and N. N. Weinberg, Phys. Rev. lett. 91, 071301 (2003).

[5] J. D. Barrow, Class. Quantom. Gravit. 21, L79 (2004); J. D. Barrow, Class. Quantom. Gravit. 21, 5619 (2004).

[6] S. Nojiri, S. Odintsov and S. Tsujikawa, Phys. Rev. D 71, 063004 (2005).

[7] M. G. Brown, K. Freese, W. H. Kinney, JCAP 0803, 002 (2008).

[8] L. Baum and P. H. Frampton, Phys. Rev. Lett. 98, 071301(2007).

[9] R. C. Tolman, Phys. Rev. 38, 1758 (1931).

[10] P. J. Steinhardt and N. Turok, Phys. Rev. D 65, 126003 (2002).

[11] X. Zhang, Eur. Phys. J. C 60, 661 (2009).

[12] R. Caldwell, Phys. Lett. B 545, 23 (2002).

[13] Y. Shtanov and V. Sahni, Phys. Lett. B 557, 1 (2003).

[14] A. Ashtekar, T. Pawlowski and P. Singh, Phys. Rev. D 74, 084003 (2006).

[15] P. Singh, K. Vandersloot and G. V. Vereshchagin, Phys. Rev. D 74, 043510 (2006).

[16] J. D. Bekenstein, Phys. Rev. D. 49, 1912 (1994).

[17] L. Susskind, J. Math. Phys. 36, 6377 (1995).

[18] A. Cohen, D. Kaplan and A. Nelson, Phys. Rev. Lett. 82, 4971 (1999).

[19] M. Li, Phys. Lett. B 603, 1 (2004).

[20] S. D. H. Hsu, Phys. Lett. B 594, 13 (2004).

[21] A. Sheykhi, Classical and Quantum Gravity, 27, 025007 (2010).

[22] I. Duran, D. Pavon, Phys. Rev. D 83, 023504 (2011).

[23] S. Nojiri and D. S. Odintsov, Gen. Rel. Grav. 38, 1285 (2006).

[24] A. Sheykhi, Phys. Lett. B 680, 113 (2009).

[25] X. Zhang, Phys. Rev. D 74, 103505 (2006).

[26] A. Sheykhi et al., Gen. Relativ. Gravit. 44, 623 (2012).

[27] M. R. Setare, M. Jamil, Europhys. Lett. 92, 49003 (2010).

[28] A. Sheykhi, Phys. Lett. B 681, 205 (2009).

[29] A. Sheykhi, Phys. Lett. B 682, 329 (2010).

[30] K. Karami, M. S. Khaledian and M. Jamil, Phys. Scr. 83, 025901 (2011)

[31] J. Shen, B. Wang, E. Abdalla, R. K. Su, Phys. Lett. B 609, 200 (2005).

[32] K. Enqvist, S. Hannestad, M. S. Sloth, JCAP 0502, 004 (2005)

[33] X. Zhang, Phys. Rev. D 79, 103509 (2009).

[34] S. M. R. Micheletti, J. Cosmol. Astropart. Phys. 4, 9
(2010).

[35] L. N. Granda, A. Oliveros, Phys. Lett. B 671, 199 (2009).

[36] M. Jamil, K. Karami, A. Sheykhi, E. Kazemi, and Z. Azarmi, Int. J. Theor. Phys. 51, 604 (2012).

[37] S. Ghaffari, M. H. Dehghani, and A. Sheykhi, Phys. Rev. D 89, 123009 (2014).

[38] C. J. Gao, X. L. Chen, and Y. G. Shen, Phys. Rev. D 79, 043511 (2009).

[39] B. Wang, E. Abdalla, F. Atrio-Barandela, and D. Pavon, Rep. Prog. Phys. 79, 096901 (2016).

[40] G. Olivares, F. Atrio, D. Pavón, Phys. Rev. D 71, 063523 (2005).

[41] O. Bertolami , F. Gil Pedro, M. Le Delliou, Phys. Lett. B 654, 165 (2007).

[42] A. A. Costa, X. D. Xu, B. Wang, E. G. M. Ferreira, and E. Abdalla, Phys. Rev. D 89, 103531 (2014).

[43] X. D. Xu, B. Wang, and E. Abdalla, Phys. Rev. D 85, 083513 (2012).

[44] J. H. He, B. Wang, and E. Abdalla, Phys. Rev. D 83, 063515 (2011).

[45] S. Wang, Y. Z. Wang, J. J. Geng, and X. Zhang, Eur. Phys. J. C 74, 3148 (2014).

[46] J. H. He, B. Wang, E. Abdallab, and D. Pavón, JCAP, 12, $022(2010)$.

[47] E. Abdalla, L. R. Abramo, and J. C. C. de Souza, Phy. Rev. D 82, 023508 (2010).

[48] X. D. Xu, B. Wang, P. Zhang, and F. A. Barandela, JCAP, 12, 001 (2013).

[49] R. G. Cai and Q. P. Su, Phys. Rev. D 81, 103514 (2010).

[50] H. Wei, Nucl. Phys. B 845, 381 (2011).

[51] H. Wei, Commun. Theor. Phys.56, 972 (2011).

[52] L. P. Chimento, Phys. Rev. D 81, 043525 (2010).

[53] L. P. Chimento, M. Forte and G. M. Kremer, Gen. Rel. Grav. 41, 1125 (2009).

[54] D. Pavón and W. Zimdahl, Phys. Lett. B 628, 206 (2005).

[55] L. Amendola, Phys. Rev. D 62, 043511 (2000).

[56] L. Amendola and C. Quercellini, Phys. Rev. D 68, 023514 (2003).

[57] L. Amendola, S. Tsujikawa, and M. Sami, Phys. Lett. B 632, 155 (2006).

[58] S. del Campo, R. Herrera, and D. Pavón, Phys. Rev. D 78, 021302 (2008).

[59] C. G. Bohmer, G. Caldera-Cabral, R. Lazkoz, and R. Maartens, Phys. Rev. D 78, 023505 (2008).

[60] S. Chen, B. Wang, and J. Jing, Phys. Rev. D 78, 123503 (2008).

[61] J. Zhang, X. Zhang, and H. Liu, Eur. Phys. J. C 52,693699 (2007).

[62] Kh. Saaidi, H. Sheikhahmadi, A. H. Mohammadi, Astro- 
phys. Space Sci. 338, 355 (2012).

[63] A. Sheykhi, M. Tavayef, M. Iran. J. Sci. Technol. Trans. Sci. doi:10.1007/s40995-016-0083-y (2016).

[64] U. Alam, V. Sahni, A. A. Starobinsky, JCAP 0406, 008 (2004);

D. Huterer, A. Cooray, Phys. Rev. D 71, 023506 (2005);
Y. G. Gong, Int. J. Mod. Phys. D 14, 599 (2005);

Y. Wang, M. Tegmark, Phys. Rev. D 71, 103513 (2005).

[65] H. C. Kim, J. W. Lee, J. Lee, Euro. Phys. Lett. 102, 29001 (2013). 